\title{
Determinação da maturidade sexual de Ucides cordatus (Crustacea, Brachyura, Ucididae) em duas áreas de manguezal do litoral sul de Pernambuco, Brasil
}

\author{
Daniela da S. Castiglioni ${ }^{1} \&$ Petrônio A. Coelho ${ }^{2}$
}

1. Núcleo de Biologia, Centro Acadêmico de Vitória, Universidade Federal de Pernambuco, Rua Alto do Reservatório, s/n, Bairro Bela Vista, 55608-680 Vitória de Santo Antão, PE, Brasil. (danielacastiglioni@yahoo.com.br)

2. Departamento de Oceanografia, Universidade Federal de Pernambuco, Av. Arquitetura s/n, Cidade Universitária, $50740-550$ Recife, PE, Brasil.

\begin{abstract}
Determination of sexual maturity of Ucides cordatus (Crustacea, Brachyura, Ucididae), in two mangrove areas of the south coast of the state of Pernambuco, Brazil. This study aims to determine the size at morphological and physiological maturity of males and females at two populations of Ucides cordatus (Linnaeus, 1763) from Tamandaré, Pernambuco, Brazil. Crabs were sampled monthly from April 2008 to March 2009 at the Ariquindá and Mamucabas mangroves by professional crab catchers during ebb tide in three replicates $\left(25 \mathrm{~m}^{2}\right.$ each, $20 \mathrm{~m}$ between plots). Crabs collected were sexed and measured (carapace width, cheliped propodus length of males and abdomen width of the fifth somite of females). Besides, the crabs were characterized according its gonad development stage. For the physiological maturity determination, crabs were arranged in two groups: juvenile crabs (specimens that showed immature and rudimentary gonads) and adult crabs (specimens with gonads at the stages classified as developing, developed, advanced or spent). The carapace width size in which $50 \%$ of males and females were considered morphologically mature was 38.0 and $35.4 \mathrm{~mm}$ (Ariquindá) and 37.3 and $32.9 \mathrm{~mm}$ (Mamucabas), respectively. Therefore, the size at physiological maturity of males and females occurred with 38.5 and $37.8 \mathrm{~mm}$ (Ariquindá) and 36.2 and $35.8 \mathrm{~mm}$, respectively (Mamucabas). Males attain morphological maturity at a higher size than females, probably due to a major investment in their somatic growth, while females spend their energy in the reproductive process. Crabs from Mamucabas mangrove attained sexual maturity at smaller sizes than Ariquindá, probably because the more impacted situation of the primer mangrove.
\end{abstract}

KEYWORDS. Crab, morphological maturity, physiological maturity.

RESUMO. Este estudo foi desenvolvido com o objetivo de determinar o tamanho da maturidade morfológica e fisiológica de machos e fêmeas em duas populações de Ucides cordatus (Linnaeus, 1763) de Tamandaré, Pernambuco, Brasil. Os caranguejos foram coletados mensalmente (abril/2008 a março/2009) nos manguezais dos rios Ariquindá e Mamucabas, por um catador, através da técnica de braceamento, durante a maré baixa em três áreas distintas de $25 \mathrm{~m}^{2}$ cada. Os caranguejos capturados foram separados por sexo e medidos (largura da carapaça, comprimento do própodo do quelípodo dos machos e largura do $5^{\circ}$ somito abdominal das fêmeas). Além disso, os caranguejos foram caracterizados em relação ao estágio de desenvolvimento gonadal. Os caranguejos com gônadas imaturas e rudimentares foram considerados jovens, enquanto os demais foram classificados como adultos (gônada em desenvolvimento, desenvolvida, avançada ou esgotada). O tamanho da largura da carapaça no qual $50 \%$ da população de $U$. cordatus foi considerada madura morfologicamente foi de 38,0 mm (machos) e 35,4 $\mathrm{mm}$ (fêmeas) em Ariquindá, enquanto para Mamucabas estes valores foram de 37,3 e $32,9 \mathrm{~mm}$, respectivamente. Na determinação da maturidade sexual fisiológica, os machos e fêmeas de Ariquindá foram considerados maduros com 38,5 e 37,8 mm, respectivamente, enquanto em Mamucabas os tamanhos obtidos foram de 36,2 e 35,8 $\mathrm{mm}$. A maturidade morfológica dos machos ocorreu com tamanho superior ao das fêmeas, provavelmente devido ao seu maior investimento em crescimento somático, enquanto as fêmeas investem mais no processo reprodutivo. Os caranguejos provenientes do manguezal de Mamucabas atingiram a maturidade sexual com tamanhos inferiores aos de Ariquindá, provavelmente devido ao maior impacto verificado para este manguezal.

PALAVRAS-CHAVE. Caranguejo, maturidade morfológica, maturidade fisiológica.

A maturidade sexual é compreendida por transformações morfológicas e fisiológicas pelas quais os jovens (ou indivíduos imaturos) adquirem a habilidade para produzir gametas e agir diretamente na estrutura populacional (Mantelatto \& Fransozo, 1996). O início da maturidade sexual está relacionado ao alcance de um determinado tamanho individual, que pode variar entre populações de uma determinada espécie e, até mesmo, entre indivíduos de uma mesma espécie (FonTELEs-FilHo, 1989).

A determinação do tamanho em que os caranguejos atingem sua maturidade sexual é um importante instrumento em estudos da dinâmica populacional e na determinação do potencial reprodutivo de uma espécie. $\mathrm{O}$ tamanho na primeira maturação em caranguejos braquiúros pode ser avaliado pela comparação entre aspectos fisiológicos (estágio de desenvolvimento das gônadas) e morfológicos externos (análise da forma e tamanho de algumas estruturas corpóreas), como analisado por Santos \& Negreiros-Fransozo (1996), López et al. (1997), Pinheiro \& Fransozo (1998), Castiglioni \& Santos (2001), Lima \& Oshiro (2006) e SiLVA et al. (2007).

Ucides cordatus (Linnaeus, 1763) é um caranguejo semiterrestre que vive somente em áreas de manguezal, ocorrendo ao longo da costa leste do Oceano Atlântico, da Flórida (EUA) ao estado de Santa Catarina, no Brasil (MeLo, 1996). Conhecido popularmente como caranguejouçá, pode atingir grande porte, sendo explorado em várias regiões brasileiras, onde serve como fonte de alimento e 
renda para populações ribeirinhas (RodRIGUEs et al., 2000; Passos \& Di Benedito, 2005; Jankowsky et al., 2006). Apesar de sua importância sócio-econômica, só recentemente tem-se notado um incremento das investigações sobre sua bioecologia (BRANCO, 1993; Botelho et al., 1999; Ivo et al., 1999, 2000; PinHeiro \& Fiscarelli, 2001; AlVes \& Nishida, 2004; Monteiro \& Coelho-Filho, 2004; Diele et al., 2005; Dalabona \& Silva, 2005; Dalabona et al., 2005; Pinheiro \& Hattori, 2006; ANDRADE et al., 2007; WundERLICH et al., 2008; ARAúJo \& CAlADO, 2009), como também por aspectos sociais, econômicos, culturais e ecológicos relacionados a sua pesca (MANeschy, 1993; Nordi, 1994; Fiscarelli \& PINHEIRO, 2002; SouTo, 2007). Com relação à determinação do tamanho da maturidade sexual que tenha levado em consideração tanto a maturidade morfológica como a fisiológica, somente um estudo foi desenvolvido por PINHEIRO \& Fiscarelli (2001) até a presente data, mas para uma população do litoral de São Paulo. No entanto, a maturidade morfológica desta espécie já foi analisada anteriormente por Dalabona et al. (2005), Pinheiro \& HATTORI (2006) e LeiTE et al. (2006), enquanto a maturidade fisiológica foi determinada por BoтеLHO et al. (1999), Ivo et al. (1999) e ANDRADE et al. (2007). Neste sentido, este trabalho tem por objetivo determinar os tamanhos de maturidade morfológica e fisiológica para $U$. cordatus, em duas áreas de manguezal do litoral sul pernambucano.

\section{MATERIAL E MÉTODOS}

As amostragens dos exemplares de Ucides cordatus foram realizadas nos manguezais do rio Ariquindá $\left(8^{\circ} 46^{\prime} 43,69^{\prime}\right.$ 'S $, 35^{\circ} 06^{\prime} 25,87^{\prime}$ 'W) e rio Mamucabas ( $\left.8^{\circ} 41^{\prime} 28,48^{\prime \prime} \mathrm{S}, 35^{\circ} 06^{\prime} 09,32^{\prime \prime} \mathrm{W}\right)$, ambos localizados no município de Tamandaré, Pernambuco. O clima da região é do tipo As' (quente e úmido), com temperaturas médias que variam entre 25 e $30^{\circ} \mathrm{C}$ e precipitação pluviométrica em torno de $2.000 \mathrm{~mm}$. Além disso, esta região possui estações bem definidas: seca (setembro a dezembro) e chuvosa (janeiro a julho) (Moura \& PassaVANTE, 1995).

O rio Ariquindá está inserido na Área de Preservação Ambiental de Guadalupe, possuindo uma extensão de $7 \mathrm{~km}$ e, juntamente com seu afluente União, detém grande importância como componente da bacia do rio Formoso (CPRH, 1999). A área de manguezal do rio Ariquindá, escolhida para a amostragem dos caranguejos, localiza-se próxima a região de sua confluência com o rio Formoso, na Praia de Carneiros, sendo o mangue composto predominantemente por Rhizophora mangle (Rhizophoraceae), seguido de Laguncularia racemosa (Combretaceae) e Avicennia spp. (Acanthaceae), esta última em menor frequência. O substrato das áreas de amostragem do caranguejo-uçá foi caracterizado como areno-lamoso.

O rio Mamucabas localiza-se quase totalmente no município de Tamandaré, Pernambuco. Este rio nasce a oeste da Reserva Biológica de Saltinho, próximo ao Engenho Barro Branco, sendo represado ao penetrar na Reserva, formando um reservatório que abastece a cidade de Tamandaré. Da nascente até a Planície Costeira, que ocorre nos arredores do núcleo urbano supracitado, o rio Mamucabas percorre sentido noroeste-sudeste, quando segue em direção sul até a desembocadura, onde se encontra com o rio Ilhetas, desaguando no Pontal que leva esse nome (CPRH, 1999). A área de amostragem de Ucides cordatus localiza-se próxima a desembocadura destes rios, sendo o mangue composto predominantemente por Laguncularia racemosa, seguido de Rhizophora mangle e, em menor abundância, Avicennia spp. O substrato das áreas de amostragens caracteriza-se como areno-lamoso.

Os caranguejos foram coletados mensalmente de abril/2008 a março/2009, por um catador de caranguejo experiente, usando a técnica de braceamento em três áreas distintas de cada manguezal $\left(25 \mathrm{~m}^{2}\right.$ cada), localizadas paralelamente ao rio e equidistantes $(20 \mathrm{~m})$. Após a amostragem, os caranguejos foram acondicionados em sacos plásticos e transportados em caixas térmicas até o laboratório, quando foram lavados, sexados e tiveram algumas de suas estruturas medidas com um paquímetro digital $(0,01 \mathrm{~mm})$ : largura da carapaça $(\mathrm{LC})$, comprimento do própodo do quelípodo maior dos machos (CPQ) e largura do $5^{\circ}$ somito abdominal das fêmeas (LA).

Por meio da remoção da carapaça e da inspeção macroscópica, o estágio de desenvolvimento gonadal de cada exemplar foi registrado, levando-se em conta aspectos como forma, tamanho e coloração. Assim, as gônadas foram classificadas de acordo com uma escala de maturidade modificada de BотеLно et al. (1999), Ivo et al. (1999) e PinHEIRO \& FisCARELLI (2001) nos seguintes estádios: machos - imaturo, gônadas indiferenciadas as quais não podem ser visualizadas macroscopicamente; rudimentar, gônada pouco visível com aspecto filamentoso e coloração transparente; em desenvolvimento, gônada visível, enovelada e de coloração esbranquiçada; desenvolvido, gônada atinge seu maior desenvolvimento, sendo bastante enovelada, de coloração branca e ocupando $1 / 2$ da cavidade cefalotorácica; esgotado, gônadas filamentosas, delgadas, flácidas e com coloração transparente; fêmeas - imaturo, gônadas indiferenciadas as quais não podem ser visualizadas macroscopicamente; rudimentar, gônadas não desenvolvidas, apresentando aspecto filamentoso, delgada e transparente; em desenvolvimento, gônadas visíveis, sendo que a coloração do ovário varia de amarelo a laranja e a relação gônada/hepatopâncreas é de aproximadamente 1/10; desenvolvido, ovário com aspecto lobulado e de coloração variando de laranja-escuro a vinho-claro e a relação gônada/hepatopâncreas é de aproximadamente 1/2; avançado, ovário ocupa toda a cavidade torácica, ultrapassando o tamanho do hepatopâncreas e apresenta aspecto lobulado mais evidente do que o estágio anterior, sendo de coloração vinho intenso; esgotado, gônadas filamentosas, delgadas e flácidas com coloração transparente a amarelo e a relação gônada/hepatopâncreas é de aproximadamente $1 / 10$.

O tamanho corpóreo médio (largura da carapaça) foi comparado entre machos e fêmeas e para cada sexo entre os diferentes manguezais pelo teste $\mathrm{t}(\alpha=0,05)$ (ZAR, 1996).

A determinação da maturidade sexual morfológica foi baseada na relação entre as estruturas CPQ vs. LC (machos) e LA vs. LC (fêmeas). A escolha destas relações foi tomada levando-se em consideração a importância das variáveis dependentes CPQ e LA no processo reprodutivo de machos e fêmeas, respectivamente. Para a determinação das categorias etárias, uma análise de $K$ - 
means clustering foi realizada, com o estabelecimento predeterminado dos grupos (jovens e adultos) e atribuição dos exemplares a eles por processo interativo que minimiza a variância dentro dos grupos e maximiza a variância entre os grupos. Após a classificação, foi aplicada uma análise discriminante bivariada, que permitiu a reclassificação destes grupos, com separação dos caranguejos em jovens e adultos. Este método estatístico foi baseado no trabalho de SAMPEDRO et al. (1999), que utilizaram um procedimento semelhante. Após a separação dos grupos, nos casos onde ocorreu sobreposição, cada categoria etária foi dividida em classes de tamanho de $5 \mathrm{~mm}$ (LC), com posterior cálculo das proporções de jovens e adultos por classe e uso da proporção de adultos para ajuste pela equação logística $\left(y=a /\left(1+b e^{-c x}\right)\right)$. Posteriormente, uma interpolação foi efetuada para a determinação do tamanho em que $50 \%$ de cada sexo se encontrava adulto. Após a divisão das categorias etárias, os dados transformados $(\log 10)$ foram submetidos a uma análise de covariância (ANCOVA), para testar os coeficientes angulares e lineares entre os grupos (jovens e adultos) $(\alpha=0,05)(\mathrm{ZAR}, 1996)$.

Para a determinação da maturidade sexual fisiológica, os machos e fêmeas foram organizados em dois grupos: caranguejos jovens, espécimes que apresentavam gônadas imaturas ou rudimentares; e caranguejos adultos, espécimes com gônadas nos estágios denominados em desenvolvimento, desenvolvidos, avançados ou esgotados. Posteriormente, os caranguejos foram agrupados em classes de tamanho (LC), com $5 \mathrm{~mm}$ de amplitude, para a obtenção da frequência de caranguejos jovens e adultos por classe. Para a análise da maturidade sexual fisiológica, foi utilizada a equação logística $\left(y=a /\left(1+b e^{-c x}\right)\right)$, como sugerido por Santos \& Negreiros-Fransozo (1996) e Pinheiro \& Fransozo (1998). O ajuste da equação foi efetuado pelo método dos mínimos quadrados (Aguillar et al., 1995; VAZZOLER, 1996).

\section{RESULTADOS}

Para a determinação do tamanho da maturidade sexual morfológica foram utilizados 1.441 exemplares, correspondendo a 639 exemplares para Ariquindá (348 machos e 291 fêmeas) e 802 para Mamucabas (430 machos e 372 fêmeas).

A largura da carapaça dos machos de Ariquindá variou de 15,1 a 79,1 $\mathrm{mm}(47,0 \pm 13,8 \mathrm{~mm})$ (média \pm desvio padrão) e das fêmeas de 9,3 a $67,9(38,6 \pm 13,3)$. No manguezal de Mamucabas, a largura da carapaça dos machos de $U$. cordatus apresentou uma amplitude de 12,9 a $79,5 \mathrm{~mm}(45,8 \pm 13,3 \mathrm{~mm})$ e nas fêmeas esta dimensão variou de 10,6 a $61,8 \mathrm{~mm}(38,8 \pm 11,4 \mathrm{~mm})$. Os machos são significativamente maiores do que as fêmeas em ambos os manguezais (Ariquindá: $\mathrm{t}=6,65$; Mamucabas: $\mathrm{t}=6,38$; $\mathrm{p}<0,05)$. No entanto, observou-se que o tamanho médio de machos e fêmeas não diferiu entre as duas populações analisadas (machos: $\mathrm{t}=1,05$ e fêmeas: $\mathrm{t}=-0,28 ; \mathrm{p}>0,05$ ).

Na determinação da maturidade sexual morfológica foram utilizados o comprimento do quelípodo dos machos e a largura do abdome das fêmeas. Nos gráficos de dispersão dos machos (CPQ vs. LC) (Figs 1,3) e fêmeas (LA vs. LC) (Figs 2, 4) percebe-se a mudança nas taxas de crescimento das variáveis dependentes em relação a largura da carapaça. No manguezal do rio Ariquindá, o tamanho (LC) no qual 50\% da população de machos $U$. cordatus foi considerada madura morfologicamente foi de $38,0 \mathrm{~mm}$ (Fig. 5), tendo o maior macho imaturo 37,36 mm e o menor maduro, 37,01 mm (Fig. 1). Já as fêmeas de Ariquindá (50\%) atingiram a maturidade morfológica aos 35,4 mm de LC (Fig. 5), tendo a maior fêmea imatura 37,19 $\mathrm{mm}$ e a menor fêmea madura $33,13 \mathrm{~mm}$ (Fig. 2). Em Mamucabas, $50 \%$ dos machos atingiram a maturidade morfológica aos 37,3 mm (Fig. 6), com o maior macho imaturo medindo $38,36 \mathrm{~mm}$ e o menor macho maduro 38,6 mm (Fig. 3). As fêmeas (50\%) foram consideradas morfologicamente maduras com 32,9 mm de LC (Fig. 6) (maior fêmea imatura $\mathrm{LC}=34,31 \mathrm{~mm}$ e menor fêmea madura $\mathrm{LC}=30,92 \mathrm{~mm}$; Fig. 4 ).

O ajuste da curva logística indicou que o tamanho da largura da carapaça em que $50 \%$ dos machos apresentam-se maduros fisiologicamente foi de 38,5 $\mathrm{mm}$ e $36,2 \mathrm{~mm}$, nos manguezais de Ariquindá e Mamucabas, respectivamente (Figs 7, 8). Tais valores para as fêmeas corresponderam a 37,8 e $35,8 \mathrm{~mm}$ de largura da carapaça (Figs 7,8), respectivamente, para estes mesmos locais.

\section{DISCUSSÃO}

Um dos mais relevantes aspectos do ciclo biológico de crustáceos é o tamanho no qual a maturidade sexual é atingida e como esta é determinada. Tradicionalmente dois critérios têm sido aplicados para se determinar este tamanho: um é baseado em aspectos diretamente relacionados à reprodução como o desenvolvimento gonadal, a análise da espermateca, a presença de fêmeas ovígeras e espermatóforos, etc. (WENNER et al., 1974, Prasad \& Neelakantan, 1990; Freire et al., 1991), enquanto o outro é baseado no surgimento dos caracteres sexuais secundários (HAEFner, 1990; CASTIGLIONI \& Negreiros-Fransozo, 2004; Dalabona et al., 2005; Pinheiro \& Hattori, 2006). Para algumas espécies de caranguejos o tamanho de maturidade segundo estes métodos não coincide (CONAN \& COMEAU, 1986; CHOY, 1988; LóPEZ-Greco \& Rodríguez, 1999). Assim, a determinação do tamanho na primeira maturação pode ser comprometida quando se leva em consideração apenas aspectos da morfologia externa. Além disso, o tamanho da maturidade sexual é geneticamente determinado para cada espécie, sofrendo influência de fatores ambientais, particularmente quando áreas geográficas distintas são comparadas (HINES, 1982).

O presente estudo confirma os resultados obtidos por Pinheiro \& Fiscarelli (2001), indicando que os tamanhos de maturidade sexual morfológica e fisiológica de machos e fêmeas de $U$. cordatus foram distintos, comprovando que o desenvolvimento gonadal não coincide com o desenvolvimento dos caracteres sexuais secundários, os quais auxiliam na cópula e na transferência das células sexuais. Esta assincronia no tamanho da maturidade sexual morfológica e fisiológica foi observada para outras espécies de braquiúros, como por exemplo, em Cyrtograpsus angulatus Dana, 1851, Armases rubripes (Rathbun, 1897) e Sesarma rectum Randall, 1840 analisados respectivamente por CASTIGLIONI 

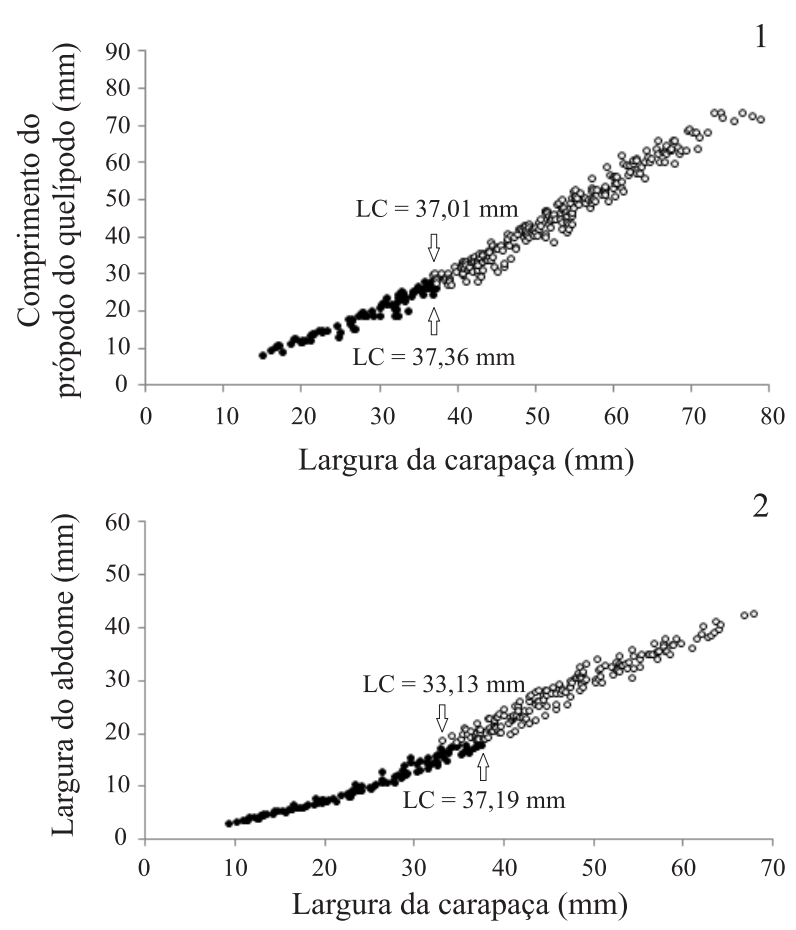

Figuras 1, 2. Dispersão das relações biométricas de Ucides cordatus (Linnaeus, 1763) no manguezal do rio Ariquindá em Tamandaré, Pernambuco: 1, entre o comprimento do própodo do quelípodo maior e largura da carapaça em machos; 2, entre a largura do abdome e a largura da carapaça em fêmeas (LC, largura da carapaça).

\& SANTOS (2001), Lima \& OshiRo (2006) e SiLVA et al. (2007). No entanto, a coincidência no tamanho de maturidade sexual morfológica e fisiológica foi observada por SANTOS \& Negreiros-Fransozo (1996) em Portunus spinimanus Latreille, 1819, por PinHEIRO \& FRANSOZO (1998) em fêmeas de Arenaeus cribrarius (Lamarck, 1818), por Сово \& Fransozo (1998, 2005) em Goniopsis cruentata (Latreille, 1803) e por Castiglioni \& Negreiros-Fransozo (2004, 2006) em U. rapax.

Pelo exposto, é muito importante a comparação de diferentes técnicas para a determinação do tamanho na maturidade sexual em crustáceos braquiúros, objetivando estimativas mais seguras. Assim, o exato tamanho estimado na maturidade deve incluir modificações expressivas na morfologia externa, além do exame do desenvolvimento gonadal, pois os tamanhos obtidos frequentemente são diferentes (GonZÁlEZ-GurRIÁRAN, 1985). De acordo com PinheIro \& Fransozo (1998), o tamanho mínimo no qual os caranguejos estão morfológica e fisiologicamente prontos para se reproduzir é denominado maturidade funcional. Se um indivíduo atinge a maturidade sexual (maturidade funcional) somente quando adquire as condições morfológicas e fisiológicas que o permita produzir células sexuais e copular com sucesso, verifica-se que o tamanho corpóreo (LC) aproximado em que tais condições são verificadas em $U$. cordatus no manguezal de Ariquindá são $38,5 \mathrm{~mm}$ em machos e 37,8 mm em fêmeas. Porém, no manguezal de Mamucabas o tamanho em que $50 \%$ dos machos e fêmeas encontram-se maduros foi de 37,3 e $35,8 \mathrm{~mm}$, respectivamente. Cabe salientar, que esses tamanhos são estimativas médias válidas para a população, embora seja possível capturar exemplares da referida espécie com tamanho na primeira maturação diferindo dessa média.
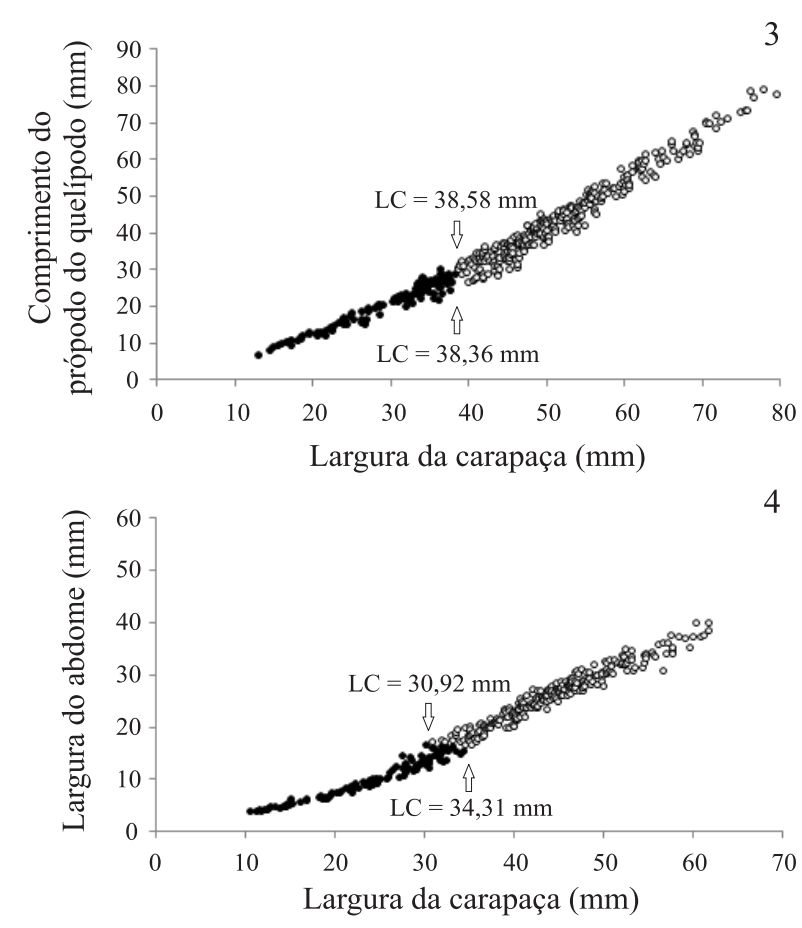

Figuras 3, 4. Dispersão das relações biométricas de Ucides cordatus (Linnaeus, 1763) no manguezal do rio Mamucabas em Tamandaré, Pernambuco: 3 , entre o comprimento do própodo do quelípodo maior e largura da carapaça em machos; 4, entre a largura do abdome e a largura da carapaça em fêmeas (LC, largura da carapaça).

O fato dos machos de Ucides cordatus maturarem com tamanho pouco superior ao das fêmeas deve-se, provavelmente, ao menor investimento energético por parte dessas últimas no crescimento. Isto ocorre por elas gastarem parte de suas energias para o propósito reprodutivo, propiciando aos machos a aquisição de tamanhos superiores com o mesmo recurso alimentar (Hartnoll, 2006). Dessa maneira, a maturidade sexual será atingida com tamanhos maiores do que nas fêmeas, como observado também por Ivo et al. (1999), PINHEIRO \& Fiscarelli (2001) e PinHeiro \& HatTori (2006) em $U$. cordatus, por CASTIGLIONI \& NEGREIROS-FRANSOZO (2004, 2006) em $U$. rapax e por Masunari \& Swiech-Ayoub (2003) em U. leptodactyla (Rathbun, 1898).

Num estudo desenvolvido com cinco espécies de braquiúros (HiNEs, 1989), verificou-se que quatro delas apresentaram variação geográfica no tamanho da maturidade sexual o que, provavelmente, pode ser causada pela temperatura ou sua interação com o fotoperíodo. Assim, variações desses fatores abióticos podem produzir clinas latitudinais nas taxas metabólicas e de crescimento somático, repercutindo em taxas mais lentas de crescimento em espécimes maiores e mais velhos, retardando a maturidade de espécies que vivem em latitudes mais altas (Hines, 1989). Jones \& Simons (1983), em seu estudo com o grapsídeo Helice crassa Dana, 1851, também observaram variação latitudinal no tamanho máximo dos caranguejos, no tamanho da maturidade das fêmeas e na produção de ovos (fecundidade), ocorrendo aumento nas maiores latitudes. Com relação à maturidade sexual de $U$. cordatus também foi observada diferença latitudinal, sendo a maturidade sexual alcançada com tamanhos inferiores nas maiores latitudes (Tab. I). Quando comparado com outras populações de Pernambuco, 


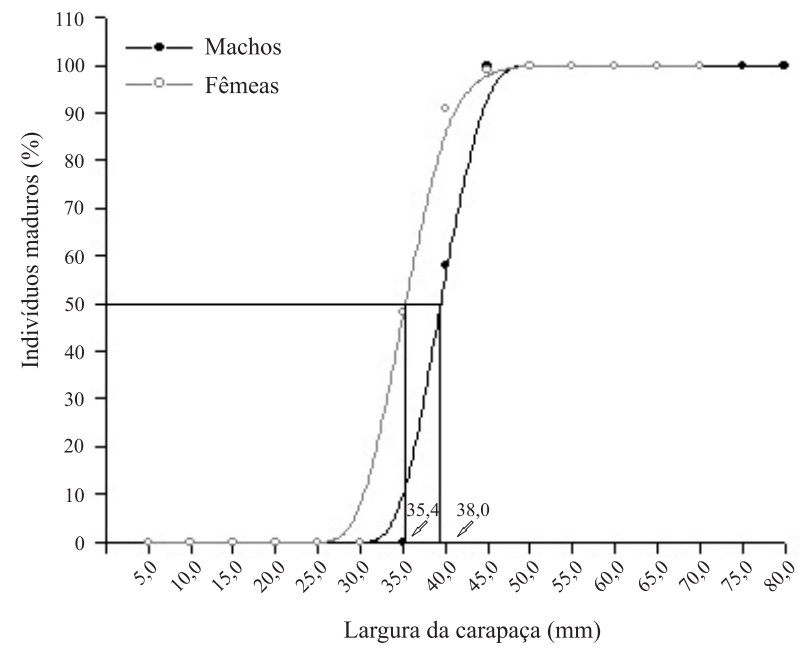

Figura 5. Curva da maturidade sexual morfológica de Ucides cordatus (Linnaeus, 1763), com estimativa do tamanho onde 50\% dos machos e fêmeas são considerados maduros morfologicamente no manguezal do rio Ariquindá em Tamandaré, Pernambuco.

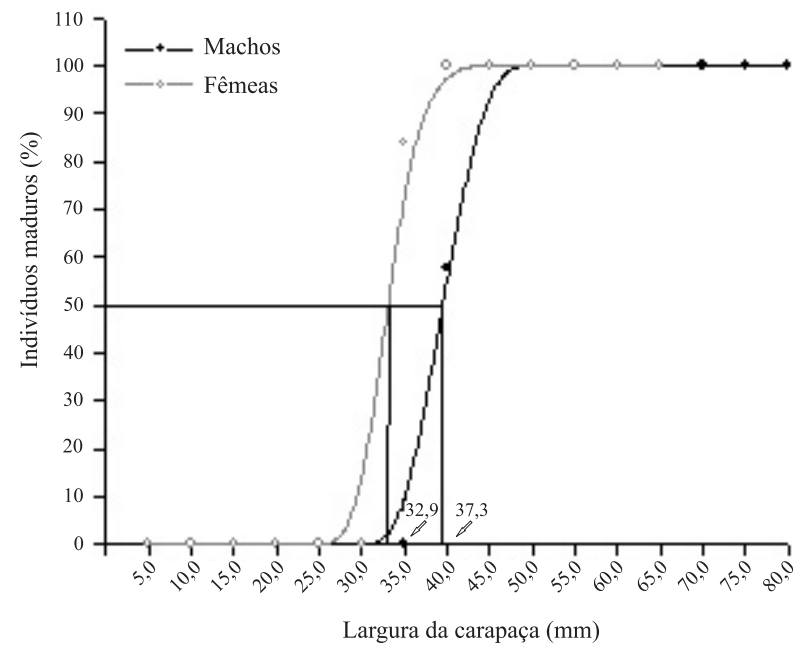

Figura 6. Curva da maturidade sexual morfológica de Ucides cordatus (Linnaeus, 1763), com estimativa do tamanho onde 50\% dos machos e fêmeas são considerados maduros morfologicamente no manguezal do rio Mamucabas em Tamandaré, Pernambuco.

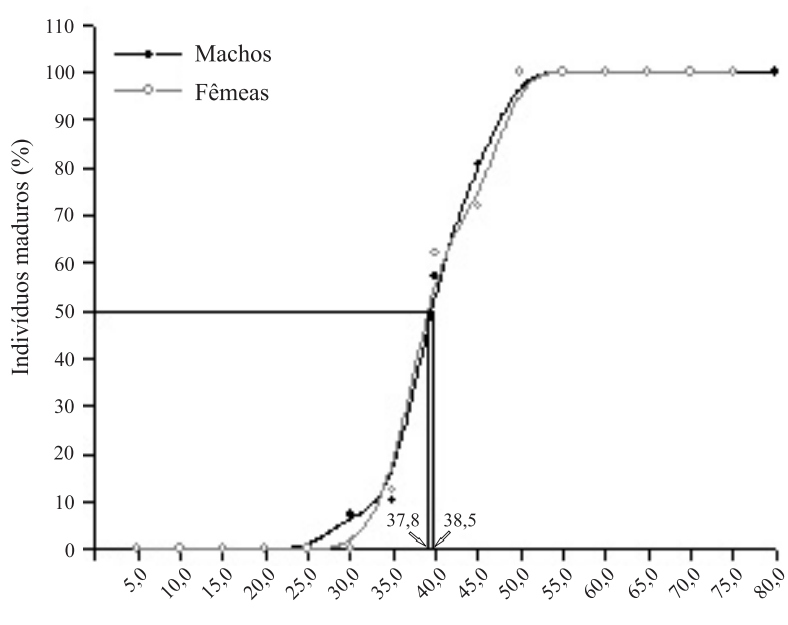

Largura da carapaça $(\mathrm{mm})$

Figura 7. Curva da maturidade sexual fisiológica de Ucides cordatus (Linnaeus, 1763), com estimativa do tamanho onde 50\% dos machos e fêmeas são considerados maduros fisiologicamente no manguezal do rio Ariquindá em Tamandaré, Pernambuco.

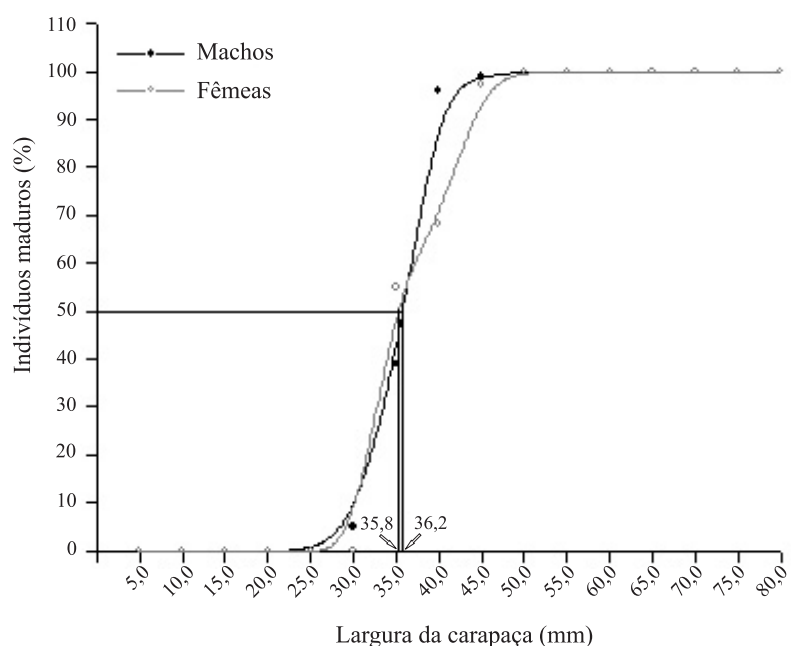

Figura 8. Curva da maturidade sexual fisiológica de Ucides cordatus (Linnaeus, 1763), com estimativa do tamanho onde 50\% dos machos e fêmeas são considerados maduros fisiologicamente no manguezal do rio Mamucabas em Tamandaré, Pernambuco.

Tabela I. Tamanho (largura da carapaça - $\mathrm{mm}$ ) da maturidade sexual morfológica e fisiológica de machos e fêmeas de Ucides cordatus (Linnaeus, 1763) em algumas localidades da costa brasileira.

\begin{tabular}{|c|c|c|c|c|c|}
\hline \multirow[t]{2}{*}{ Local } & \multicolumn{2}{|c|}{ Maturidade morfológica } & \multicolumn{2}{|c|}{ Maturidade fisiológica } & \multirow[t]{2}{*}{ Referências } \\
\hline & Machos & Fêmeas & Machos & Fêmeas & \\
\hline Ceará & & & 35,4 & 37,2 & Mota-Alves (1975) \\
\hline Rio Formoso, PE & & & 41,0 & 40,5 & Вотецно et al. (1999) \\
\hline Rio Ilhetas, PE & & & 35,5 & 38,0 & BотеLно et al. (1999) \\
\hline Rio Parnaíba, PI & & & 47,5 & 45,0 & Ivo et al. (1999) \\
\hline Laranjeiras, PR & 44,0 & 43,0 & & & DaLabona et al. (2005) \\
\hline Rio Coreaú, CE & 56,0 & & & & LeITE et al. (2006) \\
\hline Iguape, SP & 59,0 & 58,0 & & & PinHeIro \& Hattori (2006) \\
\hline Rio Curimatau, RN & & & & 41,5 & ANDRADE et al. (2007) \\
\hline Rio Ariquindá, PE & 38,0 & 35,4 & 38,5 & 37,8 & Presente estudo \\
\hline Rio Mamucabas, PE & 37,3 & 32,9 & 36,2 & 35,8 & Presente estudo \\
\hline
\end{tabular}


percebe-se que ocorre antecipação da maturidade do caranguejo-uçá, o qual se torna apto a reprodução com tamanho inferiores aos determinados por BotELHo et al. (1999). Neste sentido, tem-se a necessidade de implantar novas políticas de manejo para esta espécie na costa pernambucana, uma vez que as populações têm se reproduzido com tamanhos inferiores ao mínimo determinado pela Portaria $n^{\circ} 70 / 2000$ do Instituto Chico Mendes de Conservação da Biodiversidade (ICMBio), que é de $60 \mathrm{~mm}$.

Segundo Hines (1989), a disponibilidade de alimento, mudanças no substrato e a densidade populacional também podem regular o tamanho de maturidade, propiciando variações que modulariam diferentes padrões de crescimento, às vezes mais importantes do que parâmetros de ordem latitudinal, como observou para Hemigrapsus oregonensis (Dana, 1851) e Scyra acutifrons Dana, 1861. Se uma população vive em um ambiente rico em recursos alimentares, seus indivíduos provavelmente crescerão mais rápido, promovendo uma possível discrepância de tamanho na maturidade sexual (WENNER et al., 1974). No presente trabalho os caranguejos provenientes do manguezal de Mamucabas atingiram a maturidade sexual com tamanhos pouco inferiores aos de Ariquindá, provavelmente pelo fato deste manguezal ser mais impactado, devido à ocupação imobiliária circunvizinha às suas áreas (SANTOS et al., 2001), ocasionando maior deposição de lixo seco e desmatamento (obs. pess.). Resultados semelhantes foram observados para os caranguejos-violinistas $U$. rapax (Smith, 1870) (CASTIGLIONI \& NEGREIROS-FRANSOZO, 2004, 2006) e U. burgersi Holthuis, 1967 (BenETti \& Negreiros-Fransozo, 2004), em duas áreas distintas de manguezais do município de Ubatuba, no litoral norte paulista, nos quais os caranguejos de Itamambuca (mais ao sul) atingiram a maturidade sexual com tamanhos superiores aos de Ubatumirim (mais ao norte), provavelmente pelo fato deste apresentar um menor suprimento de matéria orgânica no sedimento disponível para a alimentação dessas espécies de caranguejosviolinistas.

Agradecimentos. A Fundação de Amparo à Ciência e Tecnologia do Estado de Pernambuco (FACEPE) pelo auxílio financeiro (APQ 0108-2.04/07) e ao CNPq pela bolsa de Desenvolvimento Científico Regional (DCR) concedida ao primeiro autor. Ao Sr. Adriano Augusto Nascimento Martins pela ajuda nos trabalhos de campo.

\section{REFERÊNCIAS BIBLIOGRÁFICAS}

Aguillar, A. T.; Malpica, Z. C. \& Urbina, B. V. 1995. Dinâmica de poblaciones de peces. Lima, Libertad. 304p.

Alves, R. R. N. \& NishidA, A. K. 2004. Population structure of the mangrove crab Ucides cordatus (Crustacea: Decapoda: Brachyura) in the estuary of the Mamanguape river, northeast Brazil. Tropical Oceanography 32(1):23-37.

Andrade, C. E.; Vasconcelos, J. A.; Oliveira, S. M. \& Ivo, C. T. 2007. Biologia e pesca do caranguejo-uçá, Ucides cordatus (Linnaeus, 1763), no estuário do Rio Curimatau, município de Canguaretama - Rio Grande do Norte. Boletim TécnicoCientífico do CEPENE 15(1):31-41.

Araújo, M. S. L. C. \& Calado, T. C. S. 2009. Bioecologia do caranguejo-Uçá Ucides cordatus (Linnaeus) no complexo estuarino lagunar Mundáu/Manguaba (CELMM), Alagoas, Brasil. Revista da Gestão Costeira Integrada 8(2):169-181.
Benetti, A. S. \& Negreiros-Fransozo, M. L. 2004. Relative growth of Uca burgersi (Crustacea, Ocypodidae) from two mangroves in the southeastern Brazilian coast. Iheringia, Série Zoologia, 94:67-72.

Botelho, E. R. O.; Dias, A. F. \& Ivo, C. T. C. 1999. Estudo sobre a biologia do caranguejo-uçá, Ucides cordatus cordatus (Linnaeus, 1763), capturado nos estuários dos rios Formoso (rio Formoso) e Ilhetas (Tamandaré), no estado de Pernambuco. Boletim Técnico-Científico do CEPENE 7(1):117-145.

Branco, J. O. 1993. Aspectos ecológicos do caranguejo Ucides cordatus (Linnaeus, 1763) (Crustacea, Decapoda) do manguezal do Itacorubi, Santa Catarina, Brasil. Arquivos de Biologia e Tecnologia 36(1):133-148.

Castiglioni, D. S. \& Negreiros-Fransozo, M. L. 2004. Comparative analysis of the relative growth of Uca rapax (Smith) (Crustacea, Ocypodidae) from two mangroves in São Paulo, Brazil. Revista Brasileira de Zoologia 21:137-144.

2006. Physiologic sexual maturity of the fiddler crab Uca rapax (Smith, 1870) (Crustacea, Ocypodidae) from two mangroves in Ubatuba, Brazil. Arquivos de Biologia e Tecnologia 49:239-248.

Castiglioni, D. S. \& Santos, S. 2001. Reproductive aspects of Cyrtograpsus angulatus Dana, 1851 (Brachyura, Grapsidae) in the Lagoa do Peixe, Rio Grande do Sul State, Brazil. Nauplius 9(1): 11-20.

CHoy, S. C. 1988. Reproductive biology of Liocarcinus puber and L. holsatus (Decapoda, Brachyura, Portunidae) from the Gower Peninsula, South Wales. Marine Ecology 9(3):227-241.

Coвo, V. J. \& Fransozo, A. 1998. Relative growth of Goniopsis cruentata (Crustacea, Brachyura, Grapsidae), on the Ubatuba region, São Paulo, Brazil. Iheringia, Série Zoologia, 84:21-28. . 2005. Physiological maturity and relationships of growth and reproduction in the red mangrove crab Goniopsis cruentata (Latreille) (Brachyura, Grapsidae) on the coast of São Paulo, Brazil. Revista Brasileira de Zoologia 22(1):219-223.

Conan, G. Y. \& Comeau, M. 1986. Functional maturity and terminal molt of male snow crab, Chionoecetes opilio. Canadian Journal of Fishery and Aquatic Science 43:1710-1719.

CPRH (Companhia Pernambucana do Meio Ambiente). 1999. Diagnóstico sócio-ambiental e ZEEC - Zoneamento Ecológico Econômico Costeiro Litoral sul de Pernambuco. Recife. 91p.

Dalabona, G. \& Silva, J. L. 2005. Período reprodutivo de Ucides cordatus (Linnaeus) (Brachyura, Ocypodidae) na Baía das Laranjeiras, sul do Brasil. Acta Biologica Paranaense 34(1, 2, 3, 4):115-126.

Dalabona, G.; Silva, J. L. \& Pinheiro, M. A. A. 2005. Size at morphological maturity of Ucides cordatus (Linnaeus, 1753) (Brachyura, Ocypodidae) in the Laranjeiras Bay, Southern Brazil. Brazilian Archives of Biology and Technology 48(1): 139-145.

Diele, K.; Koch, V. \& Saint-Paul, U. 2005. Population structure, catch composition and CPUE of the artisanally harvested mangrove crab Ucides cordatus (Ocypodidae) in the Caeté estuary, North Brazil: indication for overfishing? Aquatic Living Resources 18:169-178.

Fiscarelli, A. G. \& Pinheiro, M. A. A. 2002. Perfil sócio-econômico e conhecimento etnobiológico do catador de caranguejo-uçá, Ucides cordatus (Linnaeus, 1763), nos manguezais de Iguape (244'ㅇ), SP, Brasil. Actua Biology 24(77):129-142.

Fonteles-Filho, A. A. 1989. Recursos pesqueiros, Biologia e Dinâmica Populacional. Fortaleza, imprensa oficial do Ceará. 296p.

Freire, J.; Muiño, R.; Fernández, L. \& GonZalez-Gurriarán, E. 1991. Life cycle of Liocarcinus arcuatus (Brachyura: Portunidae) in the Ria de Arousa (Galicia, NW Spain): role of beach and mussel raft culture areas. Marine Ecology 12(3): 193-210.

González-Gurriarán, E. 1985. Reproducción de la nécora Macropipus puber (L) (Decapoda, Brachyura), y ciclo reproductivo en la Ría de Arousa (Galicia, NW España). Boletín del Instituto Español de Oceanografía 2(1):10-32. 
Haefner, Jr., P. A. 1990. Morphometry and size at maturity of Callinectes ornatus (Brachyura, Portunidae) in Bermuda. Bulletin of Marine Science 46:274-286.

Hartnoll, R. G. 2006. Reprodutive investment in Brachyura. Hydrobiologia 557:31-40.

Hines, A. H. 1982. Allometric contraints and variables of reproductive effort in Brachyura crabs. Marine Biology 69:309-320.

1989. Geographic variation in size at maturity in brachyuran crabs. Bulletin of Marine Science 45(2):356-368.

Ivo, C. T. C.; Dias, A. F.; Botelho, E. R. O.; Mota, R. I.; Vasconcelos, J. A. \& Vasconcelos, E. M. S. 2000. Caracterização das populações de caranguejo-uçá, Ucides cordatus (Linnaeus, 1763), capturados em estuários do Nordeste do Brasil. Boletim Técnico-Científico do CEPENE 8:9-43.

Ivo, C. T. C.; Dias, A. F. \& Mota, R. I. 1999. Estudo sobre biologia do caranguejo-uçá, Ucides cordatus (Linnaeus, 1763), capturado no Delta do Rio Parnaíba, Estado do Piauí. Boletim Técnico-Científico do CEPENE 7(1):53-84.

Jankowsky, M.; Pires, J. S. R. \& Nordi, N. 2006. Contribuição ao manejo participativo do caranguejo-uçá Ucides cordatus (L., 1763) em Cananéia, SP. Boletim do Instituto de Pesca 32(2):221-228

Jones, M. B. \& Simons, J. 1983. Latitudinal variation in reproductive characteristics of a mud crab Helice crassa (Grapsidae). Bulletin of Marine Science 33:656-670.

Leite, M. M. L.; Fonteles-Filho, A. A.; Silva, J. R. F. \& Cardoso, N. S. 2006. Maturidade reprodutiva funcional do caranguejo-uçá, Ucides cordatus (Crustacea, Decapoda), no estuário do Rio Coreaú, Camocim, Ceará. Boletim Técnico-Científico do CEPENE 14(2):41-49.

Lima, G. V. \& Oshiro, L. M. Y. 2006. Maturidade sexual do caranguejo Armases rubripes (Rathbun) (Crustacea, Brachyura, Sesarmidae) na Baía de Sepetiba, Rio de Janeiro, Brasil. Revista Brasileira de Zoologia 23(4):1078-1086.

López-Greco, L. S. \& Rodríguez, E. 1999. Size at the onset of sexual maturity in Chasmagnathus granulata Dana 1851 (Grapsidae, Sesarminae): a critical overall view about the usual criteria for its determination. In: SCHARAN, F. R. \& VAUPEL Klein, J. C. VON. eds. Crustacean and the biodiversity crisis. Leiden, Brill. p.675-689.

López, L. S. G.; S Tella, V. S. \& Rodríguez, E. M. 1997. Size at onset of sexual maturity in Chasmagnathus granulata (Decapoda, Brachyura). Nauplius 5(2):65-75.

Maneschy, M. C. 1993. Pescadores nos manguezais: estratégias técnicas e relações sociais de produção na captura de caranguejo. In: Furtado, L.; Leitão, W. \& Melo, A. F. eds. Povos das Águas. Realidade e perpectivas na Amazônia. Belém, Museu Paraense Emílio Goeldi. p.19-62.

Mantelatto, F. L. M. \& Fransozo, A. 1996. Size at maturity in Callinectes ornatus (Brachyura, Portunidae) from the Ubatuba Region (SP), Brazil. Nauplius 4:29-38.

Masunari, S. \& Swiech-Ayoub, B. P. 2003. Crescimento relativo de Uca leptodactyla Rathbun (Crustacea Decapoda Ocypodidae). Revista Brasileira de Zoologia 20:487-491.

Melo, G. A. S. 1996. Manual de identificação dos Brachyura (caranguejos e siris) do litoral brasileiro. São Paulo, Plêide/FAPESP. 604p.

Monteiro, B. R. \& Coelho-Filho, P. A. 2004. Estrutura populacional do caranguejo-uçá. Ucides cordatus (Linnaeus, 1763) (Crustacea, Decapoda, Ocypodidae), no estuário do Rio Paripe Itamaracá-Pernambuco. Boletim Técnico-Científico do CEPENE 12(2):101-114.

Mota-Alves, M. I. 1975. Sobre a reprodução do caranguejo-uçá,
Ucides cordatus (Linnaeus), em mangues do Estado do Ceará (Brasil). Arquivos de Ciências do Mar 15(2):85-91.

Moura, R. T. \& Passavante, J. Z. O. 1995. Biomassa fitoplanctônica na baía de Tamandaré, Rio FormosoPernambuco, Brasil. Trabalhos Oceanográficos da Universidade Federal de Pernambuco 23:1-15.

Nordi, N. 1994. A captura do caranguejo-uçá (Ucides cordatus) durante o evento reprodutivo da espécie: o ponto de vista dos caranguejeiros. Revista Nordestina de Biologia 9(1):41-47.

Passos, C. A. \& Di Beneditto, A. P. 2005. Aspectos da captura comercial do caranguejo-uçá, Ucides cordatus (L., 1763), no manguezal de Gargaú. Biotemas 18(1):223-231.

Pinheiro, M. A. A. \& Fiscarelli, A. G. 2001. Manual de apoio à fiscalização do caranguejo-uçá (Ucides cordatus). 1.ed., Itajaí, Centro de Pesquisa e Gestão dos Recursos Pesqueiros do Litoral Sudeste e Sul do Brasil/IBAMA. 43p.

Pinheiro, M. A. A. \& Fransozo, A. 1998. Sexual maturity of the speckled swimming crab Arenaeus cribrarius (Lamark, 1818) (Decapoda, Brachyura, Portunidae), in Ubatuba littoral, São Paulo State, Brazil. Crustaceana 71(4):434-452

Pinheiro, M. A. A. \& Hattori, G. 2006. Relative growth of the mangrove crab Ucides cordatus (Linnaeus, 1763) (Crustacea, Brachyura, Ocypodidae) at Iguape, São Paulo, Brazil. Brazilian Archives of Biology and Technology 49(5):813-823.

Prasad, P. N. \& Neelakantan, B. 1990. Size at maturity in the male crab Scylla serrata as determined by chela allometry and gonad condition. Fishery Technology 21(1):25-29.

Rodrigues, A. M. T.; Branco, E. J.; Saccardo, S. A. \& Blankeystein, A. 2000. Explotação do caranguejo Ucides cordatus (Decapoda: Ocypodidae) e o processo de gestão participativa para normalização da atividade na região sudeste-sul do Brasil. Boletim do Instituto de Pesca 26(1):63-78.

Sampedro, M. P.; González-Gurriarán, E.; Freire, J. \& Muiño, R. 1999. Morphometry and sexual maturity in the spider crab Maja squinado (Decapoda: Majidae) in Galicia, Spain. Journal of Crustacean Biology 19(3):578-592.

Santos, M. C. F.; Botelho, E. R. O. \& Ivo, C. T. C. 2001. Biologia populacional e manejo da pesca de aratu, Goniopsis cruentata (Latreille, 1803) (Crustacea: Decapoda: Grapsidae) no litoral sul de Pernambuco-Brasil. Boletim Técnico-Científico do CEPENE 9(1):87-123.

Santos, S. \& Negreiros-Fransozo, M. L. 1996. Maturidade fisiológica em Portunus spinimanus Latreille, 1819 (Crustacea, Brachyura, Portunidae) na região de Ubatuba, SP. Papéis Avulsos de Zoologia 39(20):365-377.

Silva, S. M. J.; Hirose, G. L. \& Negreiros-Fransozo, M. L. 2007. Population dynamic of Sesarma rectum (Crustacea, Brachyura, Sesarmidae) from a muddy flat under human impact, Paraty, Rio de Janeiro, Brazil. Iheringia, Série Zoologia, 97(2):207-214.

Souto, F. J. B. 2007. Uma abordagem etnoecológica da pesca do caranguejo Ucides cordatus Linnaeus, 1763 (Decapoda: Brachyura), no manguezal do Distrito de Acupe (Santo AmaroBA). Biotemas 20(1):69-80.

VAzzoler, A. E. A. M. 1996. Biologia reprodutiva de peixes teleósteos: teoria e prática. Maringá, EDUEM. 169p.

Wenner, A. M.; Fusaro, C. \& OAten, A. 1974. Size at onset of sexual maturity and growth rate in crustacean populations. Canadian Journal of Zoology 52(9):1095-1106.

Wunderlich, A. C; Pinheiro, M. A. A. \& Rodrigues, A. N. 2008. Biologia do caranguejo-uçá, Ucides cordatus (Crustacea: Decapoda: Brachyura), na Baía da Babitonga, Santa Catarina, Brasil. Revista Brasileira de Zoologia 25(2):188-198.

ZAR, J. H. 1996. Biostatistical analysis. New Jersey, Prentice Hall. 662p.

Recebido em setembro de 2010. Aceito em junho de 2011. ISSN 0073-4721

Artigo disponível em: www.scielo.br/isz 